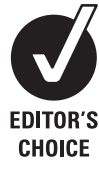

See Editorial, p 3

${ }^{1}$ The Accident and Emergency Department, Moorfields Eye Hospital NHS Foundation Trust, London, UK; ${ }^{2}$ The Control of Infection Committee, Moorfields Eye Hospital NHS Foundation Trust, London, UK; ${ }^{3}$ Department of Infection and Immunology. Guy's \& St Thomas' NHS Foundation Trust, St Thomas' Hospital, London, UK

Correspondence to: Mr J Dart, Moorfields Eye Hospital NHS Foundation Trust 162 City Road, London EC1V 2PD,UK; j.dart@ucl.ac.uk

Accepted 4 June 2008 Published Online First 12 August 2008

\title{
Identification and control of nosocomial adenovirus keratoconjunctivitis in an ophthalmic department
}

\author{
J K G Dart, ${ }^{2}$ A N El-Amir, ${ }^{1}$ T Maddison, ${ }^{2}$ P Desai, ${ }^{2}$ S Verma, ${ }^{1}$ A Hughes, ${ }^{2}$ E MacMahon ${ }^{3}$
}

ABSTRACT

Aims: To identify the extent of nosocomial adenovirus keratoconjunctivitis (AKC) and assess the effect of a new infection-control policy.

Methods: Nosocomial AKC was defined as AKC in patients attending the hospital within 3 weeks of a previous visit for an unrelated non-infective condition. An audit of culture-proven nosocomial AKC was carried out from October 1998 to September 1999 to establish its extent in our outpatient department. A new infectioncontrol policy for AKC was introduced in June 2001 that differed from the previous policy by segregating suspected AKC cases in a separate waiting area and examination room, and by expediting their examination, to reduce their exposure to both staff and patients in the common waiting areas. In October 2002, AKC was made an index infection and subjected to continual quarterly audit; the figures for this until December 2005 are reported.

Results: In the 1998-9 audit, before the introduction of the new infection-control measures, $48.4 \%$ (75/155) AKC infections were nosocomial. In the 12 months following the introduction of the quarterly audit in October 2002, $22.7 \%(5 / 22)$ were nosocomial, but thereafter the numbers of nosocomial cases dropped to $3.4 \%(8 / 235)$. Conclusion: Introduction of audit of nosocomial AKC infection identified that there was chronic cross-infection in the Moorfields Eye Hospital Accident and Emergency Department. This was controlled by the introduction of patient segregation, as an additional infection-control measure, which has almost eliminated nosocomial AKC transmission in the hospital.

Adenovirus keratoconjunctivitis (AKC), usually due to serotypes 8,19 and 37 , is the commonest nosocomial infection in eye units. ${ }^{1}$ It is caused by direct contact with contaminated multiple-use tonometer heads, dropper bottles and hospital staff's hands as well as by respiratory droplet infection and the contamination of surfaces outside the examination room ${ }^{1-3}$ (where the virus can survive for days in a desiccated state). ${ }^{4}$ AKC is of concern because of the substantial morbidity and prolonged visual complications in a proportion of cases. Hospital-acquired epidemics have led to the need to close hospital eye departments. ${ }^{5}$ The keratoconjunctivitis is often predominantly unilateral with preauricular and submandibular lymphadenopathy. Upper-respiratory-tract infection, urethritis and cervicitis may be associated. Ocular features include severe discomfort, photophobia, mucopurulent discharge, conjunctival oedema, and subconjunctival haemorrhages with punctate keratitis. Pseudomembranes can develop on the tarsal conjunctiva, usually after 7 days, and may cause conjunctival scarring. After 2 weeks, corneal subepithelial infiltrates develop which may persist for weeks, months or years, resulting in chronic discomfort, photophobia and reduced acuity. Corneal scarring is fortunately rare. Handwashing and instrument disinfection alone are insufficient in preventing nosocomial infection during an epidemic. $^{1-3}$ A prospective study by Gottsch ${ }^{5}$ showed that implementation of infection-control procedures, including a separate waiting area and examination room, reduced the incidence of epidemic and sporadic nosocomial infections in a large teaching hospital over a 4-year period.

Despite implementation of our infection-control measures including handwashing, local area surface cleaning, single-use eye-drops and avoidance of tonometry, several cases of nosocomial infection, including transmission to staff, were reported to the Infection Control Committee at Moorfields Eye Hospital in 1998. This resulted in an audit, to establish if there was an unrecognised problem of nosocomial AKC, in the hospital. The audit was followed by implementation of a new infectioncontrol policy which differed by segregating suspected AKC cases in a separate waiting area and examination room (the Red Room), and by expediting their examination, to reduce their exposure to staff and patients in the common waiting areas. The effects of these measures have been established by including nosocomial AKC as an index infection and including this in our infection-control audits.

\section{METHODS}

\section{Culture techniques}

Following collection, conjunctival swabs were placed in viral transport medium. Adenovirus was detected by shell phial culture permitting more rapid diagnosis. In brief, Vero cells grown in shell phials were inoculated with sample, centrifuged and incubated for $48 \mathrm{~h}$ prior to staining with adenovirus fluorescent antibody (Imagen) and microscopic examination. ${ }^{6}$ There was no change in sample collection, transportation or the method of viral detection over the study period (19982005)

\section{Audit strategy}

A quarterly list of all adenovirus isolates was provided by our virology service at Guy's and St Thomas' NHS Foundation Trust for the 12-month period October 1998 to September 1999. The clinical records were retrieved and examined Nosocomial AKC was defined as AKC in patients attending the hospital within 3 weeks of a previous visit for an unrelated non-infective 
condition. The incubation period is 7-10 days, and the virus may then replicate on the surface of the eye for 14 days. The period of 3 weeks was chosen to take into account both the incubation period and the period of infection with actively replicating virus. ${ }^{7}$ These cases were identified using the Hospital's computerised patient administration system and the notes retrieved for analysis. Following the introduction of the new AKC infection-control policy in June 2001, quarterly reporting of AKC isolates, and identification of nosocomial cases using the same methodology, was introduced in October 2002 when AKC was included in list of index nosocomial infections by the Hospital Control of Infection Committee. Data are reported for the audit period to October 2002 to December 2005.

\section{Alterations to the infection-control policy for suspected AKC cases}

Table 1 summarises the infection-control policies before and after the audit. The principal change in the new policy was to segregate suspected AKC cases in a separate waiting area and examination room (the Red Room), and to expedite their examination, to reduce their exposure to uninfected staff and patients in the common waiting areas.

\section{RESULTS}

\section{Audit results}

The audit results are shown in fig 1 . In the 12-month audit before the introduction of the patient-segregation policy, $48.4 \%$ of AKC cases (75/155) were nosocomial and had occurred in our Accident and Emergency Department where all acute cases are seen. Following patient segregation, the first 12-month audit of the policy showed that the nosocomial infection rate was still $22.7 \%$ (5/22), although the monthly rate was reduced (1.8 per month vs 12.9 per month). In the subsequent 27 months, only $3.4 \%(8 / 235)$ of cases were nosocomial with a monthly case rate of 8.7 .

\section{DISCUSSION}

Nosocomial adenoviral conjunctivitis is common in eye units. Prevention of epidemics is an important public health issue. Handwashing, single-dose droppers, and disinfection of tonometers and contact lenses are widely used cross-infectioncontrol measures. ${ }^{13}$ However, these measures alone may have only a limited effect, and segregation of potential cases is necessary to achieve optimal control of nosocomial disease. ${ }^{1-3} 5$ Unfortunately, segregation of potentially infected patients in outpatient clinics is harder to implement. This paper highlights how infection-control measures, designed to reduce nosocomial AKC transmission, can be further improved by the use of separate waiting room and examination rooms as described by Gottsch. ${ }^{5}$

Our rate of nosocomial infections, before implementing this policy, was $75 / 155(48.4 \%)$ in the first 12 -month period, which was audited after several staff acquired the infection in the A\&E Department. Before this audit, our hospital had depended on anecdotal reporting of an increased number of AKC cases to identify an epidemic. This audit showed that there had been a chronic epidemic of nosocomial AKC that had gone undetected by anecdotal reporting. After implementation of the patientsegregation policy, and the introduction of continuous auditing of the policy, there was a reduction in the proportion of nosocomial cases $(22.7 \%(5 / 22))$, although the total number of cases per month had also fallen, presumably due to a reduction in the prevalence of the disease in the community during this period. However, this nosocomial rate was still unacceptably high, probably reflecting incomplete implementation of the policy. In the subsequent 27 -month audit period, the nosocomial rate fell to $3.4 \%(8 / 235)$, even though the monthly rate increased overall, reflecting a rise in the incidence in the community. This improvement probably resulted from the appointment of a dedicated infection-control nurse, who worked with the A\&E staff, to increase awareness of the new

Table 1 Comparison of nosocomial adenovirus keratoconjunctivitis (AKC) infection-control policies for patients with suspected AKC

Policy for all AKC cases before the introduction of
patient segregation (until May 2001)
Policy for non-epidemic status following the introduction of patient segregation in June 2001

\section{Patient examination measures}

Single use of multidose diagnostic solutions, eg, saline Single-dose eye-drops only; all unused units discarded weekly

for fluorescein application

Avoidance of both tonometry and the use of diagnostic No change

lenses

Disinfection of tonometer heads with hypochlorite $0.1 \%$ Use of disposable tonometer heads and disinfection of non contact diagnostic lenses by washing under tap water before soaking in hypochlorite $0.1 \%$ for 5 min; contact diagnostic lenses are sterilised in gas plasma (ethylene oxide before 2005)

Handwashing with soap and water following clinical examination

Cleaning slit-lamp surfaces after clinical examination with $70 \%$ isopropyl alcohol

Patient-segregation measures

Handwashing with soap and water, hibiscrub or betadine, before and after contact with every patient

Cleaning slit-lamp surfaces before and after clinical examination with $70 \%$ isopropyl alcohol

Patients with acute conjunctivitis identified at triage in A\&E and their clinical notes marked to identify patients to staff Identified patients sent to a separate waiting area outside a designated examination cubicle (the Red Room)

Patients examined in designated cubicle and sent home as soon as a clinician is free, to reduce exposure time of the patient to common waiting and examination areas

Patient advice sheet None

Patient given advice sheet about AKC and methods of avoiding cross-infection Policy for epidemic status* following the introduction of patient segregation in June 2001

Patients with acute conjunctivitis identified at the Hospital Entrance by a trained nurse and sent to a designated waiting area before examination in the Red Room

Measures for infected doctors

Suspended from patient contact until 2 weeks after the Unchanged

onset of symptoms in the second eye

*Defined as increasing numbers of cases of nosocomial infection uncontrolled by non-epidemic measures. 


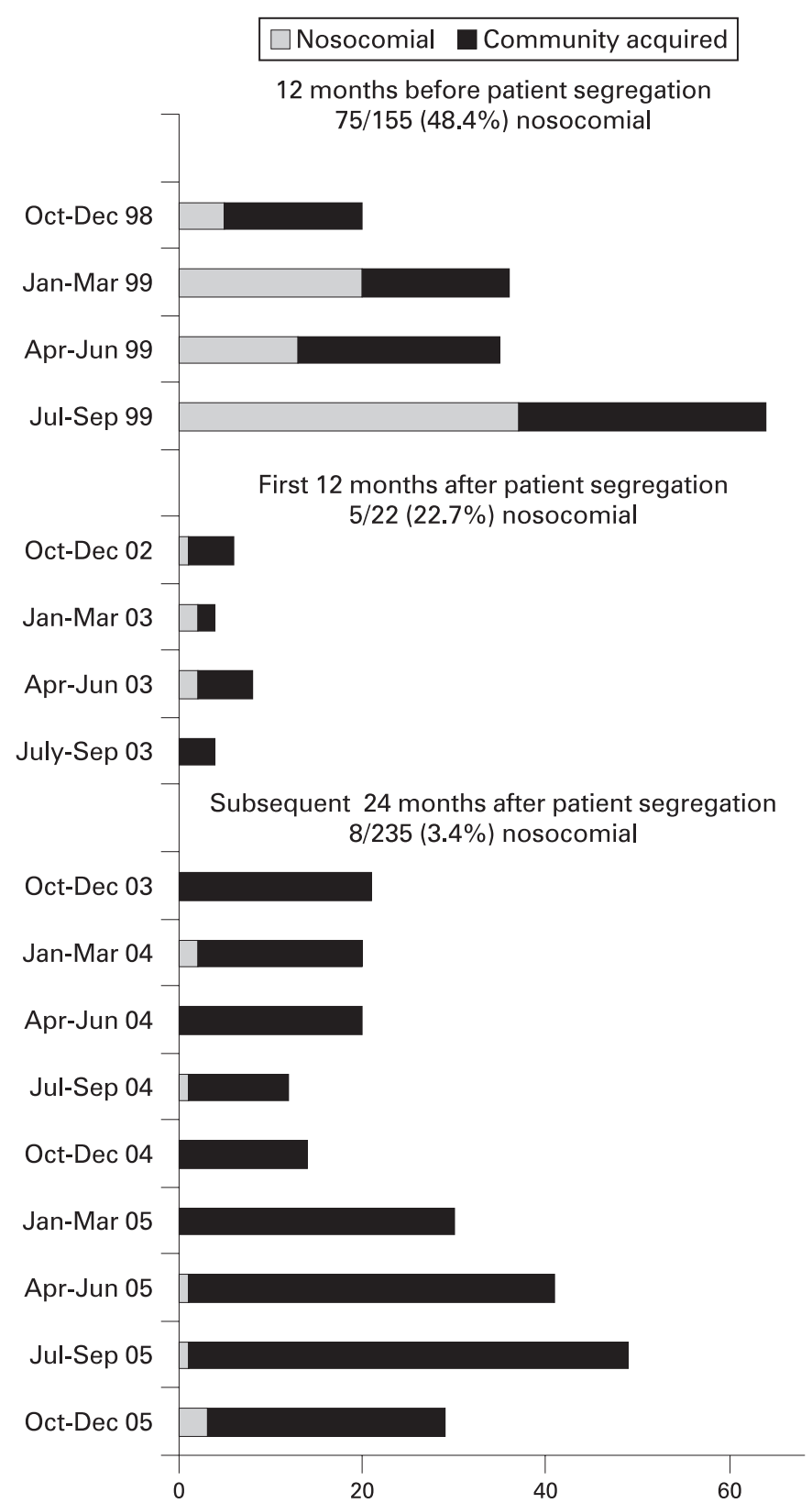

Figure 1 Audit of nosocomial and community-acquired adenovirus keratoconjunctivitis cases throughout the study period.

policy and enhance its operation. Another possible effect of the segregation policy, that cannot be measured, is that it may have resulted in better implementation of the basic hygiene measures that had constituted the previous policy, by the association of infected patients with a separate examination room. The value of the patient segregation policy is shown by the sustained improvement in the proportion of nosocomial cases throughout the last 27 months of the audit.

The implementation of this patient segregation policy for acute conjunctivitis cases has reduced nosocomial infections by more than 10-fold at our hospital. The use of the separate waiting area and examination room has been incorporated into our practice at Moorfields with education of new staff as they join the department. The inclusion of AKC as an infectioncontrol index infection, demanding ongoing quarterly audit, has ensured that the policy is implemented and remains effective.

Substantial resources were invested in providing space for the separate waiting area and examination rooms, and this may not be practical in all eye units. However, in our own unit, at times when the separate waiting area and examination room were not available (due to closure over some holiday periods), the same principles were followed with segregation of these patients and the use of a dedicated microscope and surrounding clinical area; these measures could be implemented by all eye units. Nosocomial infection is unacceptable in the current environment of healthcare provision, and strategies to curtail the rates are good practice relevant to all specialities. This paper shows how simple measures can be effective in achieving this goal.

Acknowledgements: Our gratitude to all members of staff in the Accident and Emergency Department, Audit Department and Giash Ahmed (Clinical Information Analyst); also S Palmer of the Infection and Immunology Delivery Unit (Virology Section) at Guy's and St Thomas' NHS Foundation Trust.

Funding: Moorfields Eye Hospital NHS R\&D.

Competing interests: None.

\section{REFERENCES}

1. Kennlyside RA, Hierholzer JC, D'Angelo LA. Keratoconjunctivitis associated with adenovirus 37: an extended outbreak in an ophthalmologist's office. J Infect Dis 1983;147:191-8.

2. Nauheim RC, et al. Prolonged recovery of desiccated adenovirus 19 from various surfaces. Ophthalmology 1990;97:1450-3.

3. Ford E, et al. Epidemiology of epidemic keratoconjunctivitis. Epidemiol Rev 1987:9:244-61.

4. Warren D, Nelson KE, Farrar JA, et al. A large outbreak of epidemic keratoconjunctivitis: problems in controlling nosocomial spread. J Infect Dis 1989;160:938-43.

5. Gottsch JD. Surveillance and control of epidemic keratoconjunctivitis. Trans Am Ophth Soc 1996;94:539-87.

6. Darougar $\mathbf{S}$, Walpita $\mathrm{P}$, Thaker $\mathrm{U}$, et al. Rapid culture test for adenovirus isolation. Br J Ophthalmol 1984;68:405-8.

7. Gordon YJ, Aoki K, Kinchington PR. Adenovirus keratoconjunctivitis. In: Pepose JS, Holland GN, Wilhelmus KR, eds. Ocular infection and immunity. St Louis: Mosby, 1996:877-94. 